\title{
Synthesis of New Guanidium-Meldrum Acid Zwitterionic Salts and Dynamic NMR Study of Rotational Energy Barrier Around C-NH bond of Guanidine Moiety
}

\author{
ALI AMINKHANI \\ Department of Chemistry, Khoy Branch, Islamic Azad University, Khoy, Iran \\ ${ }^{*}$ Corresponding author E-mail: ali_aminkhani@yahoo.com \\ http://dx.doi.org/10.13005/ojc/300439
}

(Received: May 15, 2014; Accepted: July 23, 2014)

\begin{abstract}
Synthesis of the Guanidium-Meldrum acid zwitterionic salts was developed through a three component reaction of 2, 2-dimethyl-1, 3-dioxane-4, 6-dione (Meldrum acid), aromatic aldehydes and $N, N, N^{\prime}, N^{\prime}$-Tetramethyl-guanidine in benzen at room temperature. These reaction conditions allow the preparation of stable zwitterionic salts in good yields. A dynamic NMR effect is observed as a result of restricted rotation around the $\mathrm{C}-\mathrm{NH}$ bond in the ${ }^{1} \mathrm{H}$ NMR spectra of these compounds. The free-energy of activation $\left(\Delta G^{\#}\right)$ for this process is $66 \pm 2 \mathrm{kJmol}^{-1}$ for $\mathbf{4 b}$.
\end{abstract}

Key words: Benzaldehydes, Dynamic NMR, Meldrum acid, Michael addition, $N, N, N^{\prime}, N^{\prime}$-Tetramethyl-guanidine, Zwitterionic salts.

\section{INTRODUCTION}

The Knoevenagel condensation of Meldrum acid with aromatic aldehydes with the aim of forming a carbon-carbon double bond is a welldocumented reaction ${ }^{1}$ that carried out using various conditions. ${ }^{2}$ Arylidene Meldrum acids are useful reactive intermediates, being susceptible to 1, 4addition, ${ }^{3}$ in Diels-Alder reactions acting as activated dienophiles ${ }^{4}$ and preparation of heterocyclic molecules such as coumarins, ${ }^{5}$ indoles and benzofurans ${ }^{6}$ and other useful compounds. ${ }^{4}, 7$ Recently we prepared an unusual chargeseparated Guanidium-meldrum acid zwitterionic salts using three component reaction. The zwitterions are often as an intermediate species in some reactions. ${ }^{8-10}$ we now describe the synthesis zwitterionic salts by means of a reaction of $N, N, N^{\prime}$, $N^{\prime}$-Tetramethyl-guanidine with conjugated electrophilic heterodienes. Dynamic NMR provides important kinetic data and affords good information in this matter on a dynamic process, when discussing the barrier separating two states that are observable by NMR spectroscopy. ${ }^{11}$ Thus, herein the free-energy of activation (" $G \#$ ) for restricted rotation around the $\mathrm{C}-\mathrm{NH}$ bond of zwitterionic salt $\mathbf{4 b}$ is described. 


\section{MATERIALAND METHODS}

\section{General}

Compounds 1-3 were obtained from Fluka and Merck and were used without further purification. The following instruments were used: mp., Electrothermal-9100 apparatus, uncorrected; IR spectra, Shimadzu IR-460 spectrometer; ${ }^{1} \mathrm{H}$ and ${ }^{13} \mathrm{C}$ NMR spectra, Bruker DRX-300 AVANC E instrument; in $\mathrm{CDCl}_{3}$ at 300.1 $\mathrm{MHz}$ and 75.4 $\mathrm{MHz}$, respectively, $\delta$ in ppm, $J$ in $\mathrm{Hz}$; El-MS (70 eV): Finnigan-MAT-8430 mass spectrometer, in $\mathrm{m} / \mathrm{z}$. Elemental analyses $(\mathrm{C}, \mathrm{H}, \mathrm{N})$ were performed with a Heraeus $\mathrm{CHN}-\mathrm{O}-$ Rapid analyzer.

General procedure for preparation of Guanidiummeldrum acid zwitterionic salts (exemplified by 4b)

To a magnetically stirred of $0.158 \mathrm{~g}$ of meldrum acid $(1.1 \mathrm{mmol})$ and $0.120 \mathrm{~g}$ of 4 methylbenzaldehyde $(1.0 \mathrm{mmol})$ in $10 \mathrm{~cm}^{3}$ of dry benzen (distilled from $\mathrm{Na} /$ benzophenone, $5.0 \mathrm{~mL}$, $0.2 \mathrm{M}$ ) was added $200 \mu \mathrm{L}$ of a $0.5 \mathrm{mM}$ solution of pyrrolidinium acetate in benzene (prepared by dropwise addition of $\mathrm{AcOH}$ to piperidine in benzene, $0.1 \mathrm{mmol}, 10 \mathrm{~mol} \%$ ) and stirred about 20 hour. Then the reaction mixture was washed with saturated $\mathrm{NaHCO}_{3}$ solution $(2 \times 10 \mathrm{~mL})$. Then $0.115 \mathrm{~g}$ of $\mathrm{N}, \mathrm{N}$, $N^{\prime}, N^{\prime}$-Tetramethyl-guanidine $(1.0 \mathrm{mmol})$ in $3 \mathrm{~cm}^{3}$ of benzen was added to organic layer over one minute at room temperature. After less four hours stirring at room temperature, the solvent was removed and the crude product washed by acetone $(2 \times 3 \mathrm{~mL})$, and residue powder filtered.

5-\{((bis (dimethylamino) methylene) ammonio) (phenyl) methyl\}-2, 2-dimethyl-4, 6-dioxo-1, 3dioxin-5-ide (4a)

White powder, mp 168-170 ${ }^{\circ} \mathrm{C}$ (decomp), $0.236 \mathrm{~g},(68 \%)$. IR $(\mathrm{KBr})\left(v_{\max } / \mathrm{cm}^{-1}\right): 1612(\mathrm{C}=\mathrm{O})$, $1681(\mathrm{C}=\mathrm{N}), 3254(\mathrm{NH}) .{ }^{1} \mathrm{H}$ NMR (300.1 MHz, $\left.\mathrm{CDCl}_{3}\right): \delta 1.56\left(6 \mathrm{H}, \mathrm{s}, \mathrm{CMe}_{2}\right), 2.92$ and $2.99(12 \mathrm{H}$, 2s, $\left.2 \mathrm{NMe}_{2}\right), 5.67\left(1 \mathrm{H}, \mathrm{d},{ }^{3} \mathrm{~J}_{\mathrm{HH}}=8.6 \mathrm{~Hz}, \mathrm{HN}-\mathrm{CH}\right), 7.20$ $\left(1 \mathrm{H}, \mathrm{t},{ }^{3} \mathrm{~J}_{\mathrm{HH}}=7.4 \mathrm{~Hz}, \mathrm{CH}\right), 7.28\left(2 \mathrm{H}, \mathrm{d},{ }^{3} \mathrm{~J}_{\mathrm{HH}}=7.4 \mathrm{~Hz}\right.$, $2 \mathrm{CH}), 7.56\left(2 \mathrm{H}, \mathrm{d},{ }^{3} \mathrm{~J}_{\mathrm{HH}}=7.4 \mathrm{~Hz}, 2 \mathrm{CH}\right), 9.05(1 \mathrm{H}, \mathrm{d}$, $\left.{ }^{3} \mathrm{~J}_{\mathrm{HH}}=8.6 \mathrm{~Hz}, \mathrm{NH}\right) \mathrm{ppm} .{ }^{13} \mathrm{C} \mathrm{NMR}\left(75.4 \mathrm{MHz} \mathrm{CDCl}_{3}\right)$ : $\delta 26.33\left(\mathrm{CMe}_{2}\right), 40.08,40.79\left(2 \mathrm{NMe}_{2}\right), 57.37$ (HN$\mathrm{CH}), 77.05$ (HC-C-C) $102.32\left(\mathrm{CMe}_{2}\right), 126.1(2 \mathrm{CH})$, $127.4(\mathrm{CH}), 128.9(2 \mathrm{CH}), 143.8(\mathrm{C}), 161.74$ (N-C-
$\mathrm{NH}), 166.66$ (2C, s, 2C=O) ppm. MS, $\mathrm{m} / \mathrm{z}(\%): 347$ $\left(\mathrm{M}^{+},<1\right), 246$ (34), 173 (100), 160 (41), 115 (79), 91 (21), 71 (52), 43 (37). Anal. Calcd for $\mathrm{C}_{18} \mathrm{H}_{25} \mathrm{~N}_{5} \mathrm{O}_{3}$ (347.41): C, 62.23; H, 7.25; N, 12.10. Found: C, 62.18; $\mathrm{H}, 7.17 ; \mathrm{N}, 12.20$

5-\{((bis (dimethylamino) methylene) ammonio) (ptolyl) methyl\}-2, 2-dimethyl-4, 6-dioxo-1, 3-dioxin5-ide (4b)

White powder, $\mathrm{mp} 182-184{ }^{\circ} \mathrm{C}$ (decomp), $0.275 \mathrm{~g},(76 \%)$. IR $(\mathrm{KBr})\left(v_{\max } / \mathrm{cm}^{-1}\right): 1608(\mathrm{C}=\mathrm{O})$, $1681(\mathrm{C}=\mathrm{N}), 3236(\mathrm{NH}) .{ }^{1} \mathrm{H}$ NMR $(300.1 \mathrm{MHz}$, $\left.\mathrm{CDCl}_{3}\right): \delta 1.55\left(6 \mathrm{H}, \mathrm{s}, \mathrm{CMe}_{2}\right), 2.28(3 \mathrm{H}, \mathrm{s}, \mathrm{Me}), 2.92$ and $2.98\left(12 \mathrm{H}, 2 \mathrm{~s}, 2 \mathrm{NMe}_{2}\right), 5.67\left(1 \mathrm{H}, \mathrm{d},{ }^{3} \mathrm{~J}_{\mathrm{HH}}=8.5\right.$ $\mathrm{Hz}, \mathrm{HN}-\mathrm{CH}), 7.08(2 \mathrm{H}, \mathrm{d}, \mathrm{J}=7.8 \mathrm{~Hz}, 2 \mathrm{CH}),, 7.38$ $(2 \mathrm{H}, \mathrm{d}, \mathrm{J}=7.8 \mathrm{~Hz}, 2 \mathrm{CH}), 8.97\left(1 \mathrm{H}, \mathrm{d},{ }^{3} \mathrm{~J}_{\mathrm{HH}}=8.5 \mathrm{~Hz}\right.$, $\mathrm{NH})$ ppm. ${ }^{13} \mathrm{C}$ NMR $\left(75.4 \mathrm{MHz}, \mathrm{CDCl}_{3}\right): \delta 21.42$ (Me), $26.34\left(\mathrm{CMe}_{2}\right), 40.07,40.73\left(2 \mathrm{NMe}_{2}\right), 57.22$ ( $\mathrm{HN}-\mathrm{CH}), 77.05$ (HC-C-C), 102.26, $\left(\mathrm{CMe}_{2}\right), 126.00$ $(2 \mathrm{CH}), 129.38(2 \mathrm{CH}), 136.56(\mathrm{C}), 140.57(\mathrm{C})$, $161.70(\mathrm{~N}-\mathrm{C}-\mathrm{NH}), 166.65$ (2C, s, 2C=O) ppm. MS, $m / z(\%): 361\left(\mathrm{M}^{+},<1\right), 246(91), 188(84), 173(97)$, 160 (30), 144 (22), 115 (100), 105 (16), 71 (45), 57 (18), 43 (34). Anal. Calcd for $\mathrm{C}_{19} \mathrm{H}_{27} \mathrm{~N}_{3} \mathrm{O}_{4}(361.44)$ : C, 63.14; H, 7.53; N, 11.63 Found: C, 63.27; H, 7.59; $\mathrm{N}, 11.58$.

5-\{((bis (dimethylamino) methylene) ammonio) (otolyl) methyl\}-2, 2-dimethyl-4, 6-dioxo-1, 3-dioxin5-ide (4c)

White powder, mp 187-189 ${ }^{\circ} \mathrm{C}$ (decomp), $0.300 \mathrm{~g},(83 \%)$. IR $(\mathrm{KBr})\left(v_{\max } / \mathrm{cm}^{\prime \prime 1}\right): 1635(\mathrm{C}=\mathrm{O})$, $1682(\mathrm{C}=\mathrm{N}), 3229(\mathrm{NH}) .{ }^{1} \mathrm{H}$ NMR $(300.1 \mathrm{MHz}$, $\left.\mathrm{CDCl}_{3}\right): \delta 1.53\left(6 \mathrm{H}, \mathrm{s}, \mathrm{CMe}_{2}\right), 2.38(3 \mathrm{H}, \mathrm{s}, \mathrm{Me}), 2.90$ and $2.95\left(12 \mathrm{H}, 2 \mathrm{~s}, 2 \mathrm{NMe}_{2}\right), 5.85\left(1 \mathrm{H}, \mathrm{d},{ }^{3} \mathrm{~J}_{\mathrm{HH}}=8.6\right.$ $\mathrm{Hz}, \mathrm{HN}-\mathrm{CH}), 7.20\left(1 \mathrm{H}, \mathrm{t},{ }^{3} \mathrm{~J}_{\mathrm{HH}}=7.4 \mathrm{~Hz}, \mathrm{CH}\right), 7.28$ $\left(2 \mathrm{H}, \mathrm{d},{ }^{3} \mathrm{~J}_{\mathrm{HH}}=7.4 \mathrm{~Hz}, 2 \mathrm{CH}\right), 7.56\left(2 \mathrm{H}, \mathrm{d},{ }^{3} \mathrm{~J}_{\mathrm{HH}}=7.4\right.$ $\mathrm{Hz}, 2 \mathrm{CH})$, 8.44. $\left(1 \mathrm{H}, \mathrm{d},{ }^{3} \mathrm{~J}_{\mathrm{HH}}=8.6 \mathrm{~Hz}, \mathrm{NH}\right) \mathrm{ppm} .{ }^{13} \mathrm{C}$ NMR (75.4 MHz, $\left.\mathrm{CDCl}_{3}\right): \delta 20.13(\mathrm{Me}), 26.23$ $\left(\mathrm{CMe}_{2}\right), 39.97,40.44\left(2 \mathrm{NMe}_{2}\right), 55.29(\mathrm{HN}-\mathrm{CH})$, 76.47 (HC-C-C) $101.96\left(\mathrm{CMe}_{2}\right), 126.09(\mathrm{CH})$, $126.45(\mathrm{CH}), 126.91(\mathrm{CH}), 130.62(\mathrm{CH}), 134.96(\mathrm{C})$, 140.77 (C), 161.45 (N-C-NH), 166.57 (2C, s, 2C=O) ppm. MS, $m / z(\%): 361\left(\mathrm{M}^{+},<1\right), 246(73), 188(87)$, 173 (94), 160 (37), 144 (19), 115 (100), 105 (9), 71 (37), 57 (23), 43 (49). Anal. Calcd for $\mathrm{C}_{19} \mathrm{H}_{27} \mathrm{~N}_{3} \mathrm{O}_{4}$ (361.44): C, 63.14; H, 7.53; N, 11.63. Found: C, 63.24; $\mathrm{H}, 7.59 ; \mathrm{N}, 11.56$. 
5-\{((bis (dimethylamino) methylene) ammonio) (m-tolyl) methyl\}-2, 2-dimethyl-4, 6-dioxo-1, 3dioxin-5-ide (4d)

White powder, $\mathrm{mp} 180-183^{\circ} \mathrm{C}$ (decomp), $0.286 \mathrm{~g},(79 \%)$. IR (KBr) $\left(v_{\max } / \mathrm{cm}^{\prime \prime 1}\right): 1612(\mathrm{C}=\mathrm{O})$, $1682(\mathrm{C}=\mathrm{N}), 3262(\mathrm{NH}) .{ }^{1} \mathrm{H}$ NMR (300.1 MHz, $\left.\mathrm{CDCl}_{3}\right): \delta 1.56\left(6 \mathrm{H}, \mathrm{s}, \mathrm{CMe}_{2}\right), 2.30(3 \mathrm{H}, \mathrm{s}, \mathrm{Me}), 2.92$ and $2.99\left(12 \mathrm{H}, 2 \mathrm{~s}, 2 \mathrm{NMe}_{2}\right), 5.67\left(1 \mathrm{H}, \mathrm{d},{ }^{3} \mathrm{~J}_{\mathrm{HH}}=8.6\right.$ $\mathrm{Hz}, \mathrm{HN}-\mathrm{CH}), 6.98-7.30(4 \mathrm{H}, \mathrm{m}, \mathrm{Ar}), 9.00\left(1 \mathrm{H}, \mathrm{d},{ }^{3} \mathrm{~J}_{\mathrm{HH}}\right.$ $=8.6 \mathrm{~Hz}, \mathrm{NH}) \mathrm{ppm} .{ }^{13} \mathrm{C} \mathrm{NMR}\left(75.4 \mathrm{MHz}, \mathrm{CDCl}_{3}\right): \delta$ $21.93(\mathrm{Me}), 26.33\left(\mathrm{CMe}_{2}\right), 40.08,40.79\left(2 \mathrm{NMe}_{2}\right)$, $57.37(\mathrm{HN}-\mathrm{CH}), 77.05(\mathrm{HC}-\mathrm{C}-\mathrm{C}) 102.32\left(\mathrm{CMe}_{2}\right)$, $123.13(\mathrm{CH}), 126.70(\mathrm{CH}), 127.85(\mathrm{CH}), 128.60$ $(\mathrm{CH}), 138.20(\mathrm{C}), 143.41(\mathrm{C}), 161.74(\mathrm{~N}-\mathrm{C}-\mathrm{NH})$, $166.66(2 \mathrm{C}, \mathrm{s}, 2 \mathrm{C}=\mathrm{O}) \mathrm{ppm}$. MS, $\mathrm{m} / \mathrm{z}(\%): 361\left(\mathrm{M}^{+},<\right.$ 1), 246 (68), 188 (73), 173 (100), 160 (29), 115 (96), 105 (22), 71 (40), 57 (16), 43 (31). Anal. Calcd for $\mathrm{C}_{19} \mathrm{H}_{27} \mathrm{~N}_{3} \mathrm{O}_{4}$ (361.44): C, 63.14; $\mathrm{H}, 7.53 ; \mathrm{N}, 11.63$. Found: C, 63.09; H, 7.47; N, 11.68 .

5-\{((bis (dimethylamino) methylene) ammonio) (4chlorophenyl) methyl\}-2, 2-dimethyl-4, 6-dioxo-1, 3-dioxin-5-ide (4e)

White powder, $\mathrm{mp} 188-190{ }^{\circ} \mathrm{C}$ (decomp), $0.267 \mathrm{~g},(70 \%)$. IR (KBr) $\left(v_{\max } / \mathrm{cm}^{-1}\right): 1612(\mathrm{C}=\mathrm{O})$, $1682(\mathrm{C}=\mathrm{N}), 3186(\mathrm{NH}) .{ }^{1} \mathrm{H}$ NMR (300.1 MHz, $\left.\mathrm{CDCl}_{3}\right): \delta 1.52\left(6 \mathrm{H}, \mathrm{s}, \mathrm{CMe}_{2}\right), 2.87$ and $2.93(12 \mathrm{H}$, 2s, $\left.2 \mathrm{NMe}_{2}\right), 5.61\left(1 \mathrm{H}, \mathrm{d},{ }^{3} \mathrm{~J}_{\mathrm{HH}}=8.7 \mathrm{~Hz}, \mathrm{HN}-\mathrm{CH}\right)$, 7.19. $(2 \mathrm{H}, \mathrm{d}, \mathrm{J}=8.4 \mathrm{~Hz}, 2 \mathrm{CH}), 7.34(2 \mathrm{H}, \mathrm{d}, \mathrm{J}=8.4$ $\mathrm{Hz}, 2 \mathrm{CH}), 8.83\left(1 \mathrm{H}, \mathrm{d},{ }^{3} \mathrm{~J}_{\mathrm{HH}}=8.7 \mathrm{~Hz}, \mathrm{NH}\right) \mathrm{ppm} .{ }^{13} \mathrm{C}$ NMR $\left(75.4 \mathrm{MHz}, \mathrm{CDCl}_{3}\right): \delta 26.33\left(\mathrm{CMe}_{2}\right), 40.02$, $40.74\left(2 \mathrm{NMe}_{2}\right), 56.82$ ( $\left.\mathrm{HN}-\mathrm{CH}\right), 77.11$ (HC-C-C), $102.29\left(\mathrm{CMe}_{2}\right), 127.53(2 \mathrm{CH}), 128.71(2 \mathrm{CH})$, 132.57 (C), 142.08 (C), 161.83 (N-C-NH), 166.46 $(2 \mathrm{C}, \mathrm{s}, 2 \mathrm{C}=\mathrm{O}) \mathrm{ppm}$. MS, $\mathrm{m} / \mathrm{z}(\%): 383\left(\mathrm{M}^{+}+2,1\right)$, $382\left(\mathrm{M}^{+}+1,1\right), 381\left(\mathrm{M}^{+}, 4\right), 368(20), 245(91), 208$ (58), 196 (39), 180 (46), 173 (100), 164 (48), 136 (74), 115 (30), 101 (30), 71 (55), 57 (31), 43 (37). Anal. Calcd for $\mathrm{C}_{18} \mathrm{H}_{24} \mathrm{ClN}_{3} \mathrm{O}_{4}$ (381.84): C, 56.62; $\mathrm{H}$, 6.34; N, 11.00. Found: C, 56.52; H, 6.44; N, 11.12.

5-\{((bis (dimethylamino) methylene) ammonio) (2chlorophenyl) methyl\}-2, 2-dimethyl-4, 6-dioxo-1, 3-dioxin-5-ide (4f)

White powder, $\mathrm{mp} 181-183^{\circ} \mathrm{C}$ (decomp), $0.275 \mathrm{~g},(72 \%)$. IR $(\mathrm{KBr})\left(v_{\max } / \mathrm{cm}^{\prime \prime 1}\right): 1608(\mathrm{C}=\mathrm{O})$, $1679(\mathrm{C}=\mathrm{N}), 3236(\mathrm{NH}) .{ }^{1} \mathrm{H}$ NMR (300.1 MHz, $\left.\mathrm{CDCl}_{3}\right): \delta 1.57\left(6 \mathrm{H}, \mathrm{s}, \mathrm{CMe}_{2}\right), 2.98$ and $3.03(12 \mathrm{H}$, 2s, $\left.2 \mathrm{NMe}_{2}\right), 5.68\left(1 \mathrm{H}, \mathrm{d},{ }^{3} \mathrm{~J}_{\mathrm{HH}}=8.7 \mathrm{~Hz}, \mathrm{HN}-\mathrm{CH}\right)$, 7.24-7.49 (4H, m, Ar), 9.00. $\left(1 \mathrm{H}, \mathrm{d},{ }^{3} \mathrm{~J}_{\mathrm{HH}}=8.7 \mathrm{~Hz}\right.$,
$\mathrm{NH})$ ppm. ${ }^{13} \mathrm{C}$ NMR (75.4 $\left.\mathrm{MHz}, \mathrm{CDCl}_{3}\right)$ :' 26.37 $\left(\mathrm{CMe}_{2}\right), 40.12,40.72\left(2 \mathrm{NMe}_{2}\right), 56.76(\mathrm{HN}-\mathrm{CH})$, 77.09 (HC-C-C), 102.25, $\left(\mathrm{CMe}_{2}\right), 125.38(\mathrm{CH})$, $125.86(\mathrm{CH}), 127.34(\mathrm{CH}), 129.56(\mathrm{CH}), 134.23$ (C), 146.07 (C), 161.95 (N-C-NH), 165.56 (2C, s, $2 \mathrm{C}=\mathrm{O}) \mathrm{ppm}$. MS, $\mathrm{m} / \mathrm{z}(\%): 383\left(\mathrm{M}^{+}+2,<1\right), 382\left(\mathrm{M}^{+}\right.$ $+1,<1), 381\left(\mathrm{M}^{+}, 2\right), 245$ (84), 208 (43), 196 (27), 180 (27), 173 (100), 164 (33), 136 (80), 115 (53), 71 (39), 57 (24), 43 (39). Anal. Calcd for $\mathrm{C}_{18} \mathrm{H}_{24} \mathrm{CIN}_{3} \mathrm{O}_{4}(381.84): \mathrm{C}, 56.62 ; \mathrm{H}, 6.34 ; \mathrm{N}, 11.00$. Found: C, 56.68; H, 6.465; N, 11.10.

5-\{((bis (dimethylamino) methylene) ammonio) (4fluorophenyl) methyl\}-2, 2-dimethyl-4, 6-dioxo-1, 3-dioxin-5-ide (4g)

White powder, mp 173-175 ${ }^{\circ} \mathrm{C}$ (decomp), $0.267 \mathrm{~g},(73 \%)$. IR $(\mathrm{KBr})\left(v_{\max } / \mathrm{cm}^{-1}\right): 1597(\mathrm{C}=\mathrm{O})$, $1682(\mathrm{C}=\mathrm{N}), 3171(\mathrm{NH}) .{ }^{1} \mathrm{H}$ NMR $(300.1 \mathrm{MHz}$, $\left.\mathrm{CDCl}_{3}\right): \delta 1.56\left(6 \mathrm{H}, \mathrm{s}, \mathrm{CMe}_{2}\right), 2.93$ and $3.00(12 \mathrm{H}$, 2s, $\left.2 \mathrm{NMe}_{2}\right), 5.68\left(1 \mathrm{H}, \mathrm{d},{ }^{3} \mathrm{~J}_{\mathrm{HH}}=8.6 \mathrm{~Hz}, \mathrm{HN}-\mathrm{CH}\right)$, $6.96(2 \mathrm{H}, \mathrm{t}, \mathrm{J}=8.7 \mathrm{~Hz}, 2 \mathrm{CH}), 7.49(2 \mathrm{H}, \mathrm{dd}, \mathrm{J}=8.7$, $5.4 \mathrm{~Hz}, 2 \mathrm{CH}), 8.96\left(1 \mathrm{H}, \mathrm{d},{ }^{3} \mathrm{~J}_{\mathrm{HH}}=8.6 \mathrm{~Hz}, \mathrm{NH}\right) \mathrm{ppm}$. ${ }^{13} \mathrm{C} \mathrm{NMR}\left(75.4 \mathrm{MHz}, \mathrm{CDCl}_{3}\right): \delta 26.32\left(\mathrm{CMe}_{2}\right), 40.12$, $40.80\left(2 \mathrm{NMe}_{2}\right), 56.88$ (HN-CH), 77.03 (HC-C-C), 102.37, $\left(\mathrm{CMe}_{2}\right), 115.44\left(\mathrm{~d},{ }^{3} \mathrm{~J}_{\mathrm{CF}}=21 \mathrm{~Hz}, 2 \mathrm{CH}\right.$,), $127.69\left(\mathrm{~d},{ }^{2} \mathrm{~J}_{\mathrm{CF}}=7.5 \mathrm{~Hz}, 2 \mathrm{CH}\right), 139.38\left(\mathrm{~d},{ }^{4} \mathrm{~J}_{\mathrm{CF}}=\right.$ $3.1 \mathrm{~Hz}, \mathrm{C}), 161.79(\mathrm{~N}-\mathrm{C}-\mathrm{NH}), 162.00\left(\mathrm{~d},{ }^{1} \mathrm{~J}_{\mathrm{CF}}=245\right.$ $\mathrm{Hz}, \mathrm{CF}), 166.56$ (2C, s, 2C=O) ppm. MS, m/z (\%): $365\left(\mathrm{M}^{+},<1\right), 245(71), 192$ (34), 173 (94), 160 (17), 148 (57), 115 (100), 71 (41), 57 (33), 43 (57). Anal. Calcd for $\mathrm{C}_{18} \mathrm{H}_{24} \mathrm{FN}_{3} \mathrm{O}_{4}$ (365.40): C, 59.17; $\mathrm{H}$, 6.62; N, 11.50 Found: C, 59.04; H, 6.54; N, 11.58.

5-\{((bis (dimethylamino) methylene) ammonio) (2fluorophenyl) methyl\}-2, 2-dimethyl-4, 6-dioxo-1, 3-dioxin-5-ide (4h) White powder, mp 176-178 ${ }^{\circ} \mathrm{C}$ (decomp), $0.296 \mathrm{~g},(81 \%)$. IR (KBr) $\left(v_{\max } / \mathrm{cm}^{-1}\right): 1620(\mathrm{C}=\mathrm{O})$, $1678(\mathrm{C}=\mathrm{N}), 3198(\mathrm{NH}) .{ }^{1} \mathrm{H}$ NMR $(300.1 \mathrm{MHz}$, $\left.\mathrm{CDCl}_{3}\right): \delta 1.68\left(6 \mathrm{H}, \mathrm{s}, \mathrm{CMe}_{2}\right), 2.89$ and $3.06(12 \mathrm{H}$, 2s, $\left.2 \mathrm{NMe}_{2}\right), 5.99\left(1 \mathrm{H}, \mathrm{d},{ }^{3} \mathrm{~J}_{\mathrm{HH}}=9.4 \mathrm{~Hz}, \mathrm{HN}-\mathrm{CH}\right)$, 6.92-7.64 (4H, m, Ar), 8.28. $\left(1 \mathrm{H}, \mathrm{d},{ }^{3} \mathrm{~J}_{\mathrm{HH}}=9.4 \mathrm{~Hz}\right.$, $\mathrm{NH})$ ppm. ${ }^{13} \mathrm{C} \mathrm{NMR}\left(75.4 \mathrm{MHz}, \mathrm{CDCl}_{3}\right): \delta 26.49$ $\left(\mathrm{CMe}_{2}\right), 39.82,40.45\left(2 \mathrm{NMe}_{2}\right), 51.38(\mathrm{HN}-\mathrm{CH})$, 72.87 (HC-C-C) $102.41\left(\mathrm{CMe}_{2}\right), 115.37\left(\mathrm{CH}, \mathrm{d},{ }^{3}\right.$ $\left.J_{\mathrm{FC}}=21.3 \mathrm{~Hz},\right), 125.2\left(\mathrm{~d},{ }^{2} \mathrm{~J}_{\mathrm{FC}}=9.4 \mathrm{~Hz}, \mathrm{CH}\right), 129.6$ $\left(d,{ }^{3} J_{F C}=22.4 \mathrm{~Hz}, \mathrm{CH}\right), 129.8\left(\mathrm{~d},{ }^{2} \mathrm{~J}_{\mathrm{FC}}=9.9 \mathrm{~Hz}, \mathrm{C}\right)$, $129.8\left(\mathrm{CH}, \mathrm{d},{ }^{4} \mathrm{~J}_{\mathrm{FC}}=3.0 \mathrm{~Hz},\right), 160.83\left(\mathrm{~d},{ }^{1} \mathrm{~J}_{\mathrm{FC}}=243.2\right.$ $\mathrm{Hz}, \mathrm{CF}) 161.01$ (N-C-NH), 167.02 (2C, s, 2C=O) ppm. MS, m/z (\%): $365\left(\mathrm{M}^{+},<1\right), 245$ (71), 173 (94), 
160 (17), 148 (57), 115 (100), 71 (41), 57 (53), 43 (57). Anal. Calcd for $\mathrm{C}_{18} \mathrm{H}_{24} \mathrm{FN}_{3} \mathrm{O}_{4}$ (365.40): C, 59.17; $\mathrm{H}, 6.62 ; \mathrm{N}, 11.50$. Found: C, 59.06; H, 6.54; N, 11.57 .

5-\{((bis (dimethylamino) methylene) ammonio) (4nitrophenyl) methyl\}-2, 2-dimethyl-4, 6-dioxo-1, 3dioxin-5-ide (4i)

Yellow powder, mp 191-193 ${ }^{\circ} \mathrm{C}$ (decomp), $0.302 \mathrm{~g},(77 \%)$. IR $(\mathrm{KBr})\left(v_{\max } / \mathrm{cm}^{-1}\right): 1612(\mathrm{C}=\mathrm{O})$, $1682(\mathrm{C}=\mathrm{N}), 3194(\mathrm{NH}) .{ }^{1} \mathrm{H}$ NMR $(300.1 \mathrm{MHz}$, $\left.\mathrm{CDCl}_{3}\right): \delta 1.55\left(6 \mathrm{H}, \mathrm{s}, \mathrm{CMe}_{2}\right), 2.92$ and $3.01(12 \mathrm{H}$, 2s, $\left.2 \mathrm{NMe}_{2}\right), 5.73\left(1 \mathrm{H}, \mathrm{d},{ }^{3} \mathrm{~J}_{\mathrm{HH}}=8.9 \mathrm{~Hz}, \mathrm{HN}-\mathrm{CH}\right)$, 7.71. $(2 \mathrm{H}, \mathrm{d}, \mathrm{J}=8.7 \mathrm{~Hz}, 2 \mathrm{CH}), 8.13(2 \mathrm{H}, \mathrm{d}, J=8.7 \mathrm{~Hz}$, $2 \mathrm{CH}), 8.92\left(1 \mathrm{H}, \mathrm{d},{ }^{3} \mathrm{~J}_{\mathrm{HH}}=8.9 \mathrm{~Hz}, \mathrm{NH}\right) \mathrm{ppm} .{ }^{13} \mathrm{C} \mathrm{NMR}$ $\left(75.4 \mathrm{MHz}, \mathrm{CDCl}_{3}\right): \delta 26.34\left(\mathrm{CMe}_{2}\right), 40.28,40.89(2$ $\mathrm{NMe}_{2}$ ), 57.11 (HN-CH), 77.07 (HC-C-C), 102.61, $\left(\mathrm{CMe}_{2}\right), 124.03(2 \mathrm{CH}), 126.78(2 \mathrm{CH}), 147.01(\mathrm{C})$, 150.69 (C), 162.12 (N-C-NH), 166.41 (2C, s, 2C=O) ppm. MS, m/z (\%): $392\left(\mathrm{M}^{+},<1\right), 245$ (100), 219 (24), 196 (92), 152 (52), 122 (5), 115 (4), 71 (22), 57 (20), 43(18). Anal. Calcd for $\mathrm{C}_{18} \mathrm{H}_{24} \mathrm{~N}_{4} \mathrm{O}_{6}$ (392.41): C, 55.09; H, 6.16; N, 14.28. Found: C, 55.22; H, 6.19; N, 14.16.

5-\{((bis (dimethylamino) methylene) ammonio) (4methoxyphenyl) methyl\}-2, 2-dimethyl-4, 6-dioxo1, 3-dioxin-5-ide (4j)

White powder, $\mathrm{mp} 169-171^{\circ} \mathrm{C}$ (decomp), $0.321 \mathrm{~g},(85 \%)$. IR $(\mathrm{KBr})\left(v_{\max } / \mathrm{cm}^{-1}\right): 1612(\mathrm{C}=\mathrm{O})$, $1682(\mathrm{C}=\mathrm{N}), 3194(\mathrm{NH}) .{ }^{1} \mathrm{H}$ NMR $(300.1 \mathrm{MHz}$, $\left.\mathrm{CDCl}_{3}\right): \delta 1.55\left(6 \mathrm{H}, \mathrm{s}, \mathrm{CMe}_{2}\right), 2.90$ and $2.96(12 \mathrm{H}$, 2s, $\left.2 \mathrm{NMe}_{2}\right), 3.74(3 \mathrm{H}, \mathrm{s}, \mathrm{OMe}), 5.63\left(1 \mathrm{H}, \mathrm{d},{ }^{3} \mathrm{~J}_{\mathrm{HH}}=\right.$ $8.4 \mathrm{~Hz}, \mathrm{HN}-\mathrm{CH}), 6.78(2 \mathrm{H}, \mathrm{d}, \mathrm{J}=8.7 \mathrm{~Hz}, 2 \mathrm{CH}),, 7.39$ $(2 \mathrm{H}, \mathrm{d}, \mathrm{J}=8.7 \mathrm{~Hz}, 2 \mathrm{CH}), 8.94\left(1 \mathrm{H}, \mathrm{d},{ }^{3} \mathrm{~J}_{\mathrm{HH}}=8.4 \mathrm{~Hz}\right.$, $\mathrm{NH})$ ppm. ${ }^{13} \mathrm{C}$ NMR $\left(75.4 \mathrm{MHz}, \mathrm{CDCl}_{3}\right): \delta 26.34$ $\left(\mathrm{CMe}_{2}\right), 40.05,40.71\left(2 \mathrm{NMe}_{2}\right), 55.62$ (OMe), 56.93 ( $\mathrm{HN}-\mathrm{CH}), 77.08$ (HC-C-C), 102.24, $\left(\mathrm{CMe}_{2}\right), 114.05$ $(2 \mathrm{CH}), 127.29(2 \mathrm{CH}), 135.91(\mathrm{C}), 158.71(\mathrm{C}), 161.62$ (N-C-NH), 166.62 (2C, s, 2C=O) ppm. MS, m/z (\%): $377\left(\mathrm{M}^{+}, 1\right), 246$ (82), 204 (19), 189 (11), 173 (100), 160 (62), 121 (19), 115 (57), 71 (34), 57 (43), 43 (31). Anal. Calcd for $\mathrm{C}_{19} \mathrm{H}_{27} \mathrm{~N}_{3} \mathrm{O}_{5}$ (377.43): C, 60.46; $\mathrm{H}, 7.21$; N, 11.13. Found: C, 60.54; H, 7.27; N, 11.06 .

5-\{((bis (dimethylamino) methylene) ammonio) (4(dimethylamino) phenyl) methyl\}-2, 2-dimethyl-4, 6-dioxo-1, 3-dioxin-5-ide (4k)

White powder, $\mathrm{mp} 123-125^{\circ} \mathrm{C}$ (decomp), $0.289 \mathrm{~g},(74 \%)$. IR ( $\mathrm{KBr})\left(v_{\max } / \mathrm{cm}^{-1}\right): 1604(\mathrm{C}=\mathrm{O})$, $1682(\mathrm{C}=\mathrm{N}), 3333(\mathrm{NH}) .{ }^{1} \mathrm{H}$ NMR $(300.1 \mathrm{MHz}$,
$\left.\mathrm{CDCl}_{3}\right): \delta 1.77\left(6 \mathrm{H}, \mathrm{s}, \mathrm{CMe}_{2}\right), 2.96$ and $2.99(12 \mathrm{H}$, 2s, $\left.2 \mathrm{NMe}_{2}\right), 3.16\left(\mathrm{Ar}-\mathrm{NMe}_{2}\right), 5.66\left(1 \mathrm{H}, \mathrm{d},{ }^{3} \mathrm{~J}_{\mathrm{HH}}=7.8\right.$ $\mathrm{Hz}, \mathrm{HN}-\mathrm{CH}), 6.68(2 \mathrm{H}, \mathrm{d}, \mathrm{J}=8.7 \mathrm{~Hz}, 2 \mathrm{CH}), 8.27(2 \mathrm{H}$, $\mathrm{d}, \mathrm{J}=8.7 \mathrm{~Hz}, 2 \mathrm{CH}), 8.93\left(1 \mathrm{H}, \mathrm{d},{ }^{3} \mathrm{~J}_{\mathrm{HH}}=7.8 \mathrm{~Hz}, \mathrm{NH}\right)$ ppm. ${ }^{13} \mathrm{C}$ NMR $\left(75.4 \mathrm{MHz}, \mathrm{CDCl}_{3}\right): \delta 26.34\left(\mathrm{CMe}_{2}\right)$, 39.81, $40.48\left(2 \mathrm{NMe}_{2}\right), 41.05\left(\mathrm{Ar}-\mathrm{NMe}_{2}\right) 56.90(\mathrm{HN}-$ $\mathrm{CH}), 77.01$ (HC-C-C), 102.19, $\left(\mathrm{CMe}_{2}\right), 112.92$ $(2 \mathrm{CH}), 127.06(2 \mathrm{CH}), 131.69(\mathrm{C}), 149.88(\mathrm{C}), 161.52$ (N-C-NH), 166.78 (2C, s, 2C=O) ppm. MS, m/z (\%): $390\left(\mathrm{M}^{+},<1\right), 246$ (89), 217 (21), 173 (94), 160 (31), 142 (16), 115 (100), 89 (27), 71 (42), 57 (24), 43 (42). Anal. Calcd for $\mathrm{C}_{20} \mathrm{H}_{30} \mathrm{~N}_{4} \mathrm{O}_{4}$ (390.23): C, 61.52; $\mathrm{H}, 7.74 ; \mathrm{N}, 14.35$. Found: C, 61.60; H, 7.84; N, 14.39.

5-\{((bis (dimethylamino) methylene) ammonio) (4bromophenyl) methyl\}-2, 2-dimethyl-4, 6-dioxo-1, 3-dioxin-5-ide (4I)

White powder, mp 204-206 ${ }^{\circ} \mathrm{C}$ (decomp), $0.324 \mathrm{~g},(76 \%)$. IR $(\mathrm{KBr})\left(v_{\max } / \mathrm{cm}^{-1}\right): 1613(\mathrm{C}=\mathrm{O})$, $1682(\mathrm{C}=\mathrm{N}), 3193(\mathrm{NH}) .{ }^{1} \mathrm{H}$ NMR $(300.1 \mathrm{MHz}$, $\left.\mathrm{CDCl}_{3}\right)$ : ' $1.574\left(6 \mathrm{H}, \mathrm{s}, \mathrm{CMe}_{2}\right), 2.89$ and $2.96(12 \mathrm{H}$, 2s, $\left.2 \mathrm{NMe}_{2}\right), 5.62\left(1 \mathrm{H}, \mathrm{d}^{3} \mathrm{~J}_{\mathrm{HH}}=8.7 \mathrm{~Hz}, \mathrm{HN}-\mathrm{CH}\right), 7.38$ $(4 \mathrm{H}, \mathrm{s}, \mathrm{Ar}) 8.87\left(1 \mathrm{H}, \mathrm{d},{ }^{3} \mathrm{~J}_{\mathrm{HH}}=8.7 \mathrm{~Hz}, \mathrm{NH}\right) \mathrm{ppm} .{ }^{13} \mathrm{C}$ $\operatorname{NMR}\left(75.4 \mathrm{MHz}, \mathrm{CDCl}_{3}\right): \delta 26.35\left(\mathrm{CMe}_{2}\right), 40.05$, $40.81\left(2 \mathrm{NMe}_{2}\right), 56.91$ (HN-CH), 77.07 (HC-C-C), 102.36, $\left(\mathrm{CMe}_{2}\right), 120.73(\mathrm{C}), 127.91(2 \mathrm{CH}), 131.69$ $(2 \mathrm{CH}), 142.57(\mathrm{C}), 161.87(\mathrm{~N}-\mathrm{C}-\mathrm{NH}), 166.50(2 \mathrm{C}$, $\mathrm{s}, 2 \mathrm{C}=\mathrm{O}) \mathrm{ppm}$. MS, $\mathrm{m} / \mathrm{z}(\%): 427\left(\mathrm{M}^{+},<1\right), 425\left(\mathrm{M}^{+}\right.$, < 1), 325 (14), 246 (58), 253 (21), 173 (89), 209 (19), 160 (31), 115 (100), 71 (29), 57 (24), 43 (38). Anal. Calcd for $\mathrm{C}_{18} \mathrm{H}_{24} \mathrm{BrN}_{3} \mathrm{O}_{4}$ (426.30): C, 61.52; $\mathrm{H}$, 7.74 ; N, 14.35. Found: C, 61.60; H, 7.81; N, 14.39.

\section{RESULTSAND DISCUSSION}

The three component condensation reactions of Meldrum acid $\mathbf{1}$, benzaldehyde derivatives 2, and $N, N, N^{\prime}, N^{\prime}$-Tetramethyl-guanidine 3 proceeded with piperidinium acetate at room temperature in benzene and were complete within about a day. Analysis of the IR, ${ }^{1} \mathrm{H}$ and ${ }^{13} \mathrm{C}$ NMR spectroscopy, and mass spectrometry spectra as well as elemental analysis confirm the formation and isolation of the desired zwitterion salts as shown in scheme $\mathbf{1}$. The IR spectrum of $\mathbf{4 b}$ exhibits a $\mathrm{NH}$ stretching band at $3236 \mathrm{~cm}^{-1}$, signals for carbonyl groups at $1601 \mathrm{~cm}^{-1}$ and for $\mathrm{C}=\mathrm{N}$ at 1682 $\mathrm{cm}^{-1}$. The ${ }^{1} \mathrm{H}$ NMR spectrum of $\mathbf{4 b}$ exhibited four single sharp lines readily recognized as $\mathrm{CMe}_{2}(\delta=$ $1.55 \mathrm{ppm}), \operatorname{Ar}-\mathrm{Me}(\delta=2.28 \mathrm{ppm})$, two $\mathrm{NMe}_{2}$ groups 
$(\delta=2.92,2.98 \mathrm{ppm})$, along with two characteristic doublets $\left(\delta=5.67\right.$ and $\left.8.97 \mathrm{ppm},{ }^{3} \mathrm{~J}_{\mathrm{HH}}=8.5\right)$ for the
$\mathrm{H}-\mathrm{C}-\mathrm{NH}$ moiety. All aromatic protons resonate at $\delta$ $=7.05-7.38$ ppm.<smiles>CC1(C)OC(=O)CC(=O)O1</smiles><smiles>CN(C)C(=N)N(C)C=O</smiles>

2 piperidinium acetate $10 \mathrm{~mol} \%$

Benzen

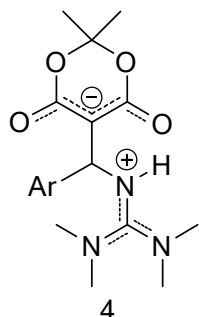

4

\begin{tabular}{l|l|l}
2,4 & $\mathrm{Ar}$ & Yield(\%) of 4 \\
\hline a & $\mathrm{Ph}$ & 68 \\
b & p-Tolyl & 76 \\
c & o-Tolyl & 83 \\
d & m-Tolyl & 79 \\
e & $4-\mathrm{CIC}_{6} \mathrm{H}_{4}$ & 70 \\
$\mathrm{f}$ & $2-\mathrm{CIC}_{6} \mathrm{H}_{4}$ & 72 \\
g & $4-\mathrm{FC}_{6} \mathrm{H}_{4}$ & 73 \\
$\mathrm{~h}$ & $2-\mathrm{FC}_{6} \mathrm{H}_{4}$ & 81 \\
i & $4-\mathrm{NO}_{2} \mathrm{C}_{6} \mathrm{H}_{4}$ & 77 \\
j & $4-\mathrm{OMeC}_{6} \mathrm{H}_{4}$ & 85 \\
k & $4-\mathrm{N}, \mathrm{N}_{-} \mathrm{diMeC}_{6} \mathrm{H}_{4}$ & 74 \\
l & $4-\mathrm{BrC}_{6} \mathrm{H}_{4}$ & 76
\end{tabular}

\section{Scheme 1: Synthesis of Guanidium-Meldrum acid zwitterionic salts}

The ${ }^{1} \mathrm{H}$-decoupled ${ }^{13} \mathrm{C}$ NMR spectrum of 4b showed six distinct signals below 100 ppm, which were readily recognized as arising from $\mathrm{Ar}$ Me $(\delta=21.42 \mathrm{ppm}), \mathrm{CMe}_{2}$ groups $(\delta=26.34 \mathrm{ppm})$, two $\mathrm{NMe}_{2}$ groups $(\delta=40.06,40.73 \mathrm{ppm})$, methine $(\delta=57.22,77.05 \mathrm{ppm})$ carbon atoms. The meldrum acid residue showed only one signal for the carbonyl ( $\delta=166.65$ ) group, which is consistent with the presence of a local $C_{\mathrm{s}}$ symmetry, which exhibited characteristic signals with appropriate chemical shifts. The ${ }^{1} \mathrm{H}$ and ${ }^{13} \mathrm{C}$ NMR spectra of the other compounds are similar to those of $\mathbf{4 b}$ except for the aryl groups, which exhibit characteristic signals with appropriate chemical shifts. The mass and elemental analyses data were in agreement with the proposed structures. The formation of zwitterionic salts 4 can be rationalized by initial formation of a conjugated electron-deficient heterodyne 5 through Knoevenagel condensation ${ }^{8}$ of the 1 and benzaldehydes 2 using piperidinium acetate followed by a Michael-type 9,11 addition reaction with $N, N, N^{\prime}, N^{\prime}$-Tetramethyl-guanidine 3 to afford compound 4 as shown in scheme 2. We didn't detect any cyclization of synthetic compounds at room temperature, ${ }^{12}$ refluxing for $5 \mathrm{~h}$ and microwave irradiation.

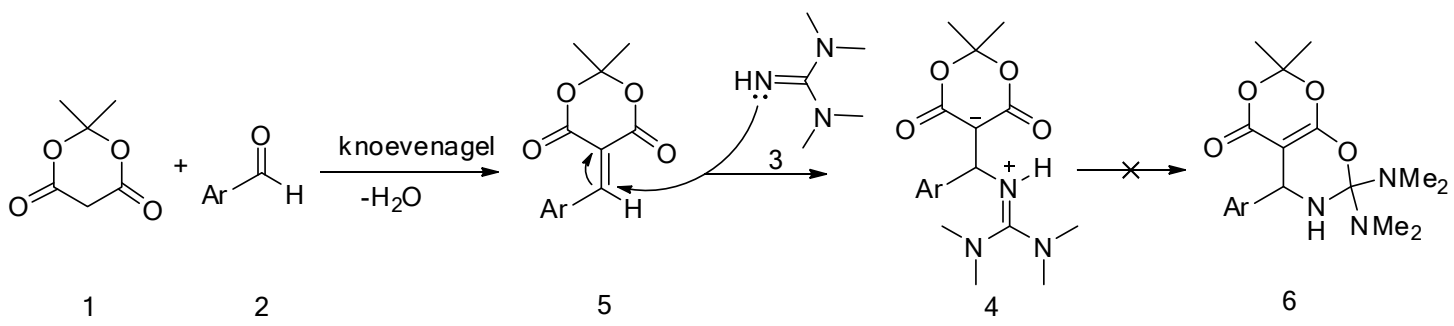

Scheme 2: Proposed mechanism for the formation of Guanidium-Meldrum acid zwitterionic salts 
The ${ }^{1} \mathrm{H}$ NMR spectrum of $\mathbf{4 b}$ in $\mathrm{CDCl}_{3}$ at ambient temperature displayed two single resonances due to the $\mathrm{NMe}_{2}(\delta=2.96,3.02)$ protons. At about $+30^{\circ} \mathrm{C}$, the resonances arising from the $\mathrm{NMe}_{2}$ protons were appreciably broadened when compared to the corresponding signals at room temperature, whereas other group's resonances remained unchanged. The $\mathrm{NMe}_{2}$ protons coalescences near $+35^{\circ} \mathrm{C}$ and appeared as a fairly symmetrical line at $+40^{\circ} \mathrm{C}$. The variable temperature spectra allowed calculating the free-energy barrier for the C-NH bond two $\mathrm{NMe}_{2}$ groups rotation in $\mathbf{4 b}$ (Scheme 3). Using the expression $\mathrm{k}=v \Delta v / 2$, first order rate constant $\left(\mathrm{k}=39.96 \mathrm{~s}^{-1}\right)$ was calculated for the $\mathrm{N}-\mathrm{C}$ bond rotation in $\mathbf{4 b}$ at $308 \mathrm{~K}$ as shown in Table. Application of the absolute rate theory with a transmission coefficient of $\mathbf{4 b}$ gave free-energy activation $\left(\Delta G^{\#}\right)$ of $66 \pm 2 \mathrm{k} \mathrm{Jmol}^{-1}$, where all known sources of errors were estimated and included. ${ }^{14}$ Only two 1:1 singlets are observed for the four diastereotopic methyl groups of the guanidino moiety in the ${ }^{1} \mathrm{H}$ and ${ }^{13} \mathrm{C}$ NMR spectra of $4 \mathrm{~b}$ at room temperature. Two rotational processes, i) $\mathrm{C}-\mathrm{NH}$ bond rotation (A, B) and ii) $\mathrm{C}-\mathrm{NMe}_{2}$ bond rotation (C), can be envisaged in the guanidino moiety of 4 . When both of these processes are fast on the NMR timescale, the four Me groups become equivalent and exhibit a single resonance, as for the spectra<smiles>CC(NC(=O)N(C)C)C(C(=O)N(C)C)C(Br)C1C(=O)OC(C)(C)OC1=O</smiles>

A<smiles>CN(C)C(=O)N(C)C(Br)C1OC(=O)OC(C)(C)OC1=O</smiles>

B<smiles>CN(C)C(=NC(=O)C1OC(=O)C(=O)OC(C)(C)O1)N(C)C</smiles>

C

Scheme 3: Dynamic effect of synthesized zwitterionic salts

Table 1: Selected ${ }^{1} \mathrm{H}$ chemical shifts ( $300 \mathrm{MHz}$ ) and activation parameters of $4 \mathrm{~b}$ in $\mathrm{CDCl}_{3}$

\begin{tabular}{|c|c|c|c|c|c|}
\hline $\operatorname{Temp}\left({ }^{\circ} \mathrm{C}\right)$ & Resonance $\left(\mathrm{NMe}_{2}\right)$ & $\Delta \mathrm{v}(\mathrm{Hz})$ & $\mathrm{K}\left(\mathrm{S}^{-1}\right)$ & $\mathrm{Tc}(\mathrm{K})$ & $\Delta \mathbf{G}^{\#}\left(\mathrm{KJmol}^{-1}\right)$ \\
\hline 35 & 2.99 & - & - & 308 & - \\
\hline 30 & $2.96 \quad 3.02$ & 18 & 39.96 & - & $66 \pm 2$ \\
\hline
\end{tabular}

observed above $308 \mathrm{~K}$. two observed signals for these groups' shows one of the processes should be fast on the NMR timescale. We conceder the contribution of the valence-bond structures $A$ and $B$ to the resonance hybrid of 4 is in fact more important than that of $C$ for the simple reason that they correspond to a more highly substituted carbonnitrogen double bond. ${ }^{14}$ Therefore the faster process can be the $\mathrm{C}-\mathrm{NH}$ rotation (process i). In summary, we have reported the synthesis of guanidinium zwitterionic salts by an efficient and simple approach along with three component condensation in benzene in presence of piperidinium acetate at room temperature within one day. A dynamic NMR effect is observed in the ${ }^{1} \mathrm{H}$ NMR spectra of these compounds as a result of restricted rotation around $\mathrm{C}-\mathrm{NH}$ bond for two $\mathrm{NMe}_{2}$ groups.

\section{ACKNOWLEDGMENTS}

Author gratefully acknowledges financial support from the Research Council of Khoy Branch Islamic Azad University. 


\section{REFERENCES}

1. a) McNab, H. Chem. Soc. Rev., 1978, 7, 345358.

b) Chen, B. C. Heterocycles, 1991, 32, 529597.

c) Dumas. A. N.; Fillion, E. Acc. Chem. Res., 2010, 43, 440-454.

2. a) Ayoubi, S. A.; Texier-Boullet, F.; Hamelin, J. Synthesis, 1994, 258-260.

b) Ayoubi, S. A.; Texier-Boullet, F. J. Chem. Res. (S), 1995, 205-206.

c) Jones, G. Organic Reactions, vol. 15, Wiley, New York, 1967, p 204.

d) Choudary, B. M.; Kantam, M. L.; Neeraja, V.; Rao, K. K.; Figueras, F.; Delmotte, L. Green Chem., 2001, 3, 257-260.

e) Narsaiha, A. V.; Basak, A. K.; Visali, B.; Nagaiah, K. Synth Commun., 2004, 34, 2893-2901.

f) Desai, U. V.; Pore, D. M.; Mane, R. B.; Solabanavar, S. B.; Wadgaonkar, P. P. Synth. Commun. 2004, 34 (1), 25-32.

g) Fildes, D.; Caignaert, V.; Villemin, D.; Jaffres, P. Green Chem. 2001, 3, 52-56.

h) Rao, P. S.; Venkataratnam, R. V. Indian J. Chem. B, 1993, 32, 484-486.

i) Dumas, A. M.; Seed, A.; Zorzitto, A. K.; Fillion, E. Tetrahedron Letters, 2007, 48, 7072-7074.

j) Kraus G. A.; Krolski, M. E. J. Org. Chem. 1986, 51, 3347-3350.

k) Thorat, M. T.; Jagdale, M. H.; Mane, R. B.; Salunkhe, M. M.; Wadgaonkar, P. P. Curr. Sci, 1987, 56 (15), 771-772.

I) Bigi, F.; Carloni, S.; Ferrari, L.; Maggi, R.; Mazzacani, A.; Sartori, G. Tetrahedron Lett. 2001, 42, 5203-5205.

m) Hedge, A.; Kruse, C. W.; Snyder, H. R. J. Org. Chem. 1961, 26, 3166-3170. n) Darvatkar, N. B.; Deorukhkar, A. R.; Bhilare, S. V.; Salunkhe, M. M. Synthetic Communications, 2006, 36, 3043-3057.

3. a) Wilsily, A.; Fillion, E. J. Org. Chem. 2009, 74, 8583-8594.

b) Knopfel, T. F.; Zarotti, P.; Ichikawa, T.; E. Carreira, M. J Am. Chem. Soc. 2005, 127, 9682-9683.

c) Ziegler, F. E.; Guenther, T.; Nelson, R. V. Synth. Commun. 1980, 10, 661-665.

4. a) Patasz, A.; Jelska, K.; Ozóg, M.; Serda, P. Monatsh. Chem. 2007, 138, 481-488.

b) Brown, R. C. F.; McMullen, G. L. Aust. J. Chem. 1974, 27, 2385-2391.

5. Mahulikar, P. P.; Mane, R. B. J. Chem. Res. 2006, 4, 15-18.

6. Baxter, G. J.; Brown, R. F. C.; McMullen, G. L. Aust. J. Chem. 1974, 27, 2605-2610.

7. Brown, R. C. F.; Eastwood, F. W., Harrington, K. J. Aust. J. Chem. 1974, 27, 2373-2384.

8. Fan, M. J.; Qian, B.; Zhao, L. B.; Liang, Y. M. Tetrahedron, 2007, 63, 8987-8992.

9. Esmaeili, A. A.; Zendegani, H. Tetrahedron, 2005, 61, 4031-4034.

10. Nair, V.; Unni Vinod, A.; Abhilash, N.; Menon, R. S.; Santhi, V.; Luxmi Varma, R.; Viji, S.; Mathewa, S.; Srinivas, R. Tetrahedron, 2003, 59, 10279-10286.

11. Yavari, I.; Aminkhani A.; Arab-Salmanabadi, S. Monatsh. Chem. 2012, 143, 1195-1198.

12. Yavari; I.; Shahvelayatia; A. Malekafzalia, A. Journal of Sulfur Chemistry 2010, 31(6), 499-508.

13. Oki, M.; Application of Dynamic NMR Spectroscopy to Organic Chemistry, $\mathrm{VCH}$ publishers, Deerfield. Beach, Florida, 1985.

14. Yavari, I. Roberts, J. D.; Biochem Biophys Commun, 1978, 83, 635-640. 